Provided for non-commercial research and education use. Not for reproduction, distribution or commercial use.

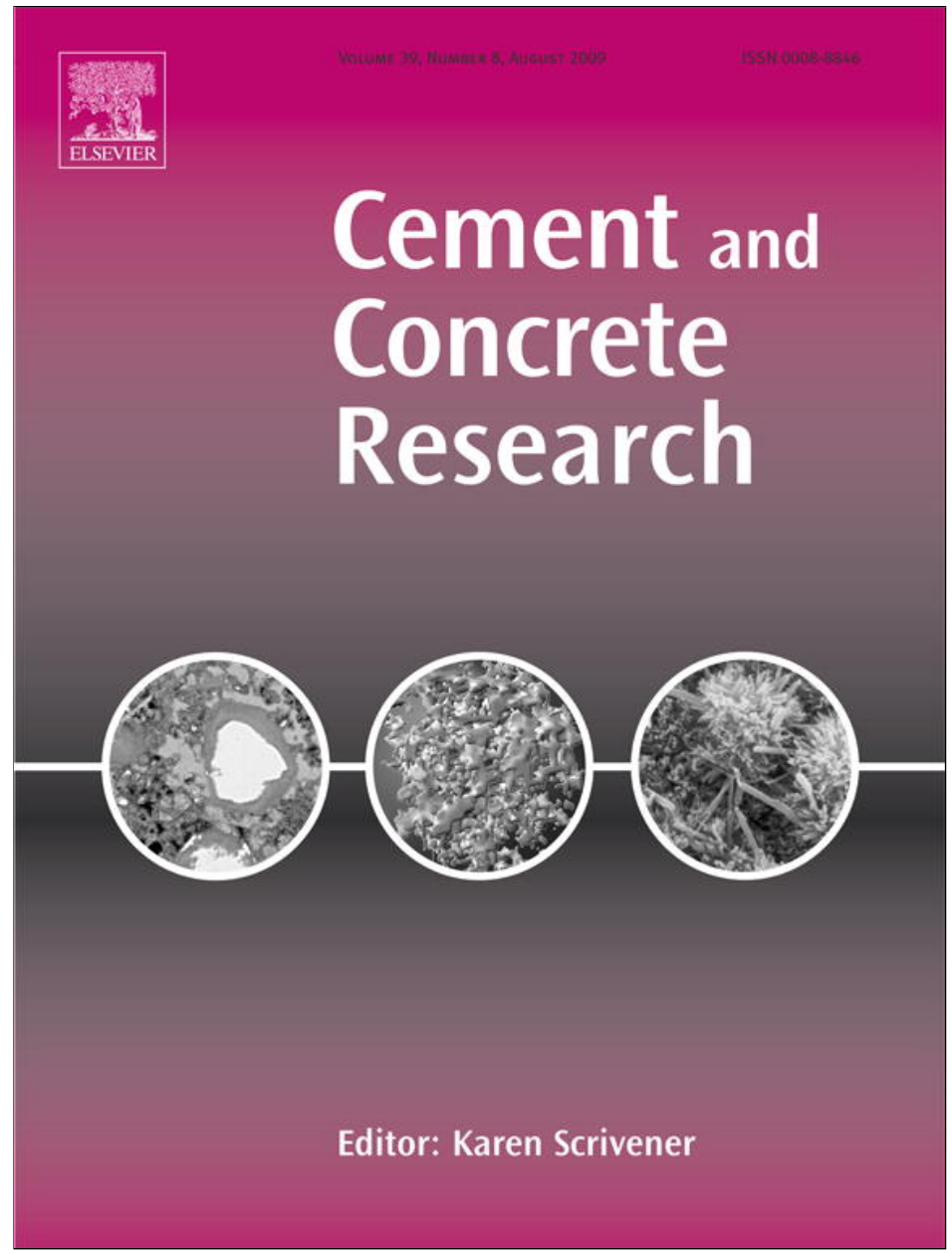

This article appeared in a journal published by Elsevier. The attached copy is furnished to the author for internal non-commercial research and education use, including for instruction at the authors institution and sharing with colleagues.

Other uses, including reproduction and distribution, or selling or licensing copies, or posting to personal, institutional or third party websites are prohibited.

In most cases authors are permitted to post their version of the article (e.g. in Word or Tex form) to their personal website or institutional repository. Authors requiring further information regarding Elsevier's archiving and manuscript policies are encouraged to visit:

http://www.elsevier.com/copyright 


\title{
Adsorption of superplasticizer admixtures on alkali-activated slag pastes
}

\author{
M. Palacios ${ }^{\text {a, } *}$, Y.F. Houst ${ }^{\text {b }}$, P. Bowen ${ }^{\text {b }}$, F. Puertas ${ }^{\text {a }}$ \\ ${ }^{a}$ Eduardo Torroja Institute for Construction Science (CSIC). Box 19002, 28080 Madrid, Spain \\ b Laboratoire de technologie des poudres. École Polytechnique Fédérale de Lausanne (EPFL) 1015 Lausanne, Switzerland
}

\section{A R T I C L E I N F O}

\section{Article history:}

Received 2 April 2008

Accepted 14 May 2009

\section{Keywords:}

Alkali-activated slag cement

Admixtures

Portland cement

Adsorption

Rheology

\begin{abstract}
A B S T R A C T
Alkali-activated slag (AAS) binders are obtained by a manufacturing process less energy-intensive than ordinary Portland cement (OPC) and involves lower greenhouse gasses emission. These alkaline cements allow the production of high mechanical strength and durable concretes. In the present work, the adsorption of different superplasticizer admixtures (naphthalene-based, melamine-based and a vinyl copolymer) on the slag particles in AAS pastes using alkaline solutions with different $\mathrm{pH}$ values have been studied in detail. The effect of the superplasticizers on the yield stress and plastic viscosity of the AAS and OPC pastes have been also evaluated.

The results obtained allowed us to conclude that the adsorption of the superplasticizers on AAS pastes is independent of the $\mathrm{pH}$ of the alkaline solutions used and lower than on OPC pastes. However, the effect of the admixtures on the rheological parameters depends directly on the type and dosage of the superplasticizer as well as of the binder used and, in the case of the AAS, on the pH of the alkaline activator solution. In 11.7-pH $\mathrm{NaOH}-\mathrm{AAS}$ pastes the dosages of the superplasticizers required to attain similar reduction in the yield stress are ten-fold lower than for Portland cement. In this case the superplasticizers studied show a fluidizing effect considerably higher in 11.7-pH NaOH-AAS pastes than in OPC pastes. In 13.6-pH NaOH-AAS pastes, the only admixture observed to affect the rheological parameters is the naphthalene-based admixture due to its higher chemical stability in such extremely alkaline media.
\end{abstract}

(C) 2009 Elsevier Ltd. All rights reserved.

\section{Introduction}

In recent years the construction industry has devoted great efforts to develop cements which have lower environmental impact than Portland cement: lower emissions of greenhouse gasses ( primarily $\mathrm{CO}_{2}$ ) emitted and lower energy expended in manufacture. One of the alternatives studied is the development of clinker-free cements, such as alkaliactivated slag (AAS) cements. These binders are obtained by mixing granulated blast furnace slag, an iron industry by-product, with high alkaline solutions. Many papers [1-5] have been written attesting to the high mechanical strength and durability of these materials, which are comparable in both respects to ordinary Portland cement (OPC).

The use of high-range water reducing admixtures, or superplasticizers, to improve rheology and workability of OPC concretes has become a common practice. Such admixtures not only improve the in situ placement of concrete, but lower the water demand by up to $40 \%$, improving mechanical and durable properties [6,7]. The mechanisms involved and the superplasticizer compatibility with Portland cement have been studied and described in numerous articles [8-10]. These papers report that such admixtures adsorb onto the surface of cement particles, causing inter-particle electrostatic and electrosteric repulsion. In the resulting dispersion, the water retained in the flocs is

\footnotetext{
* Corresponding author.

E-mail address: martapalacios@ietcc.csic.es (M. Palacios).
}

released, enhancing flowability. Other authors have described the factors affecting the fluidizing effect of superplasticizer admixtures, concluding that it depends not only on the type of admixture used, but also on factors associated with the cement itself, such as $C_{3} A$ content, type and content of calcium sulphate used as a setting regulator [1113], alkalis content [14], specific surface and particle size distribution $[9,15]$ and type of mineral additions $[10,16]$.

By contrast, very few studies have been conducted on the effect of superplasticizers on AAS cements and the mechanisms involved are unknown $[17,18]$. These papers show that the fluidizing effect of superplasticizers in AAS cements differs from the effect on OPC mixes and clearly appears to depend on the type of admixture added and, in particular, on the nature of the alkaline activator solution. Prior research $[18,19]$ has concluded that while superplasticizers based on polycarboxylate, melamine, vinyl copolymer and naphthalene show significant fluidizing properties in OPC systems. However, in waterglass or NaOH-AAS these systems lose their fluidizing properties due to their chemical instability in highly alkaline solutions. However, naphthalene-based admixture is chemically stable in $\mathrm{NaOH}$ solution and keeps its fluidizing properties allowing a $14 \%$ decrease in the liquid/solid (1/s) ratio employed in the preparation of $\mathrm{NaOH}-\mathrm{AAS}$ mortars and produces a significant rise in the mechanical strength $[18,19]$.

While certain superplasticizers are known to have beneficial effects on AAS system flowability, and the mechanism is expected to 
Table 1

Chemical composition of blast furnace slag and Portland cement.

\begin{tabular}{|c|c|c|c|c|c|c|c|c|c|c|c|c|}
\hline$\%$ & $\mathrm{CaO}$ & $\mathrm{SiO}_{2}$ & $\mathrm{Al}_{2} \mathrm{O}_{3}$ & $\mathrm{MgO}$ & $\mathrm{Fe}_{2} \mathrm{O}_{3}$ & $\mathrm{SO}_{3}$ & $\mathrm{~S}^{2-}$ & $\mathrm{Na}_{2} \mathrm{O}$ & $\mathrm{K}_{2} \mathrm{O}$ & $\mathrm{CaO}$ free & L.O.I. & I.R. \\
\hline Blast furnace slag & 41.37 & 34.95 & 13.11 & 7.12 & 0.69 & 0.04 & 1.92 & 0.27 & 0.23 & - & 2.02 & 0.11 \\
\hline Portland cement & 62.52 & 18.86 & 5.50 & 2.17 & 3.29 & 3.33 & - & 0.15 & 0.90 & 1.34 & 3.28 & 0.29 \\
\hline
\end{tabular}

L.O.I.: loss on ignition.

I.R.: insoluble residue.

be similar to OPC, the mechanisms underlying and adsorption of these admixtures on AAS has yet to be studied in detail and verified. The present study will measure adsorption isotherms of three superplasticizers on AAS and OPC suspensions. The effect of the superplasticizers on zeta potential and rheological behaviour of both binders and eventual similarities and differences will be highlighted. Also the effect of the $\mathrm{pH}$ of the alkaline solution employed in AAS pastes will be investigated.

\section{Experimental}

\subsection{Materials used}

The chemical composition of the Spanish ground granulated blast furnace slag and Portland cement type CEM I 42.5R used are given in Table 1. Table 2 shows the mineralogical composition of the cement. The slag has a vitreous phase content of $99 \%$ and a specific surface of $325 \mathrm{~m}^{2} / \mathrm{kg}$, while the specific surface of the Portland cement was $347 \mathrm{~m}^{2} / \mathrm{kg}$.

The blast furnace slag was activated with $\mathrm{NaOH}$ solutions at pH 11.7 and 13.6.

The commercial superplasticizer admixtures used were:

- melamine formaldehyde derivative (M)

- naphthalene formaldehyde derivative (NF)

- vinyl copolymer (V).

Chemical structure of the admixtures is shown in Fig. 1 and physical and chemical characterization of the superplasticizers is given in Table 3.

\subsection{Tests conducted}

2.2.1. Determination of superplasticizer adsorption isotherms on AAS and OPC suspensions

The methodology used to determine the superplasticizer adsorption isotherms on AAS and OPC suspensions was similar to that used by Perche [20]. Two solutions of $\mathrm{NaOH}$ with different $\mathrm{pH}$ values, 11.7 and 13.6, were used to prepare the AAS suspensions. These alkaline solutions were prepared using $\mathrm{NaOH}$ in pellet form and their $\left[\mathrm{OH}^{-}\right]$were $0.005 \mathrm{M}$ and $2.57 \mathrm{M}$, respectively. Suspensions were prepared mixing $10 \mathrm{~g}$ of slag and $18 \mathrm{~g}$ of $\mathrm{NaOH}$ solution for $30 \mathrm{~min}$ at $25^{\circ} \mathrm{C} .2 \mathrm{ml}$ of water containing dosages of from 0 to $40 \mathrm{mg}$ of superplasticizer/g of slag were then added to the mix, which was stirred for a further $30 \mathrm{~min}$. The suspensions were subsequently centrifuged for $3 \mathrm{~min}$, after which the supernatant was filtered through a $0.45 \mu \mathrm{m}$ filter and neutralized with acetic acid.

The amount of admixture in the liquid phase and therefore not adsorbed by the slag was determined by ultraviolet spectroscopy (UV) using a Perkin Elmer spectrometer model Lamda 900 with wavelength settings of 267, 288 and $250 \mathrm{~nm}$ for admixtures M, NF and V, respectively. After the determination of the calibration curves, the

Table 2

Mineralogical composition of Portland cement after Bogue.

\begin{tabular}{lllll}
\hline$\%$ & $\mathrm{C}_{3} \mathrm{~S}$ & $\mathrm{C}_{2} \mathrm{~S}$ & $\mathrm{C}_{3} \mathrm{~A}$ & $\mathrm{C}_{4} \mathrm{AF}$ \\
\hline Portland cement & 65.04 & 5.01 & 9.01 & 10.01 \\
\hline
\end{tabular}

amount of admixture adsorbed on the slag was taken as the difference between the initial amount of admixture and the amount present in the supernatant after adsorption.

The same tests were conducted on OPC pastes to compare the differences in adsorption between the two types of materials.

\subsubsection{Determination of the effect of superplasticizers on the zeta} potential of AAS and OPC suspensions

The effect of different dosages of superplasticizer on the zeta potential of 11.7-pH NaOH-AAS and OPC was determined with a Colloidal Dynamics Acoustosizer IIs apparatus. This test could not be conducted with 13.6-pH NaOH-activated slag suspensions because the extreme alkalinity of the suspension would have damaged the electroacustosizer instrument used.

Slag and OPC suspensions were prepared by mixing $40 \mathrm{~g}$ of binder having a particle size smaller than $20 \mu \mathrm{m}$ (approximately the upper size limit for zeta potential measurement using electroacustics) with $160 \mathrm{~g}$ of alkaline solution and water, respectively, (solid fraction in the suspension $=0.222$ ). Suspensions were stirred for $15 \mathrm{~min}$ in a magnetic stirrer, they were then placed in a sonicator for $5 \mathrm{~min}$ and immediately after in the measuring cell to determine its zeta potential. Dosages of M, NF and V ranging from 0 to $40 \mathrm{mg}$ superplasticizer/g binder were automatically added to these suspensions.

2.2.3. Study of the effect of admixtures on AAS and OPC paste rheology

Both the rheological behaviour of AAS and OPC pastes and the effect of different dosages of $\mathrm{M}, \mathrm{NF}$ and $\mathrm{V}$ on that behaviour were determined. The dosages ranged from 0 to $4.20 \mathrm{mg}$ superplasticizer $/ \mathrm{g}$ binder, being the range recommended by the admixtures producers.

AAS and OPC pastes were prepared by mixing with a spatula $100 \mathrm{~g}$ of binder in solutions containing the respective percentages of admixture. Table 4 shows the liquid/solid ( $1 / \mathrm{s})$ ratio used to prepare the pastes. The $1 / \mathrm{s}$ used to obtain workable pastes was higher in 13.6- a)<smiles>CCCCNc1nc(NC[AsH3])nc(NCCOCC)n1</smiles>

c)

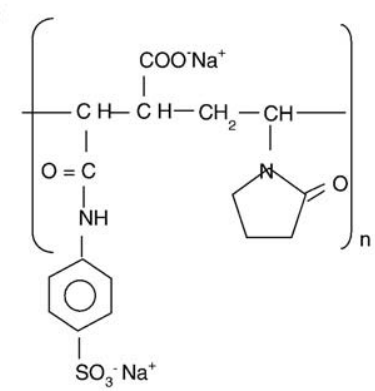

Fig. 1. Chemical structures of the superplasticizers: a) melamine derivate, b) naphthalene derivate, c) vinyl copolymer. 
Table 3

Physical and chemical characteristics of superplasticizers.

\begin{tabular}{llll}
\hline Admixture & Melamine-based & Naphthalene-based & Vinyl copolymer \\
\hline Solids content (\%) & 40 & 40 & 25 \\
$\mathrm{pH}$ & 8.22 & 7.86 & 6.80 \\
Density $\left(\mathrm{g} / \mathrm{cm}^{3}\right)$ & 1.23 & 1.20 & 1.14 \\
$M_{\mathrm{w}}$ of the main peaks & $M_{\mathrm{w} 1}=16,000$ & $M_{\mathrm{w} 1}=3200$ & $M_{\mathrm{w} 1}=28,000$ \\
$\quad$ determined by GPC $(\mathrm{kD})$ & $M_{\mathrm{w} 2}=3600$ & & $M_{\mathrm{w} 2}=14000$ \\
Intrinsic viscosity $(\mathrm{ml} / \mathrm{g})$ & 8.63 & 6.09 & 52.87 \\
$\% \mathrm{C}$ & 29.03 & 46.59 & 34.05 \\
$\mathrm{Na}(\mathrm{ppm})$ & 39350 & 42400 & 38950 \\
$\mathrm{~K}(\mathrm{ppm})$ & 2930 & 320 & 160 \\
\hline
\end{tabular}

Table 4

Liquid/solid ratio employed in the preparation of AAS and OPC pastes.

\begin{tabular}{ll}
\hline Paste & Liquid/solid \\
\hline $11.7-\mathrm{pH} \mathrm{NaOH}-\mathrm{AAS}$ & 0.40 \\
13.6-pH NaOH-AAS & 0.50 \\
OPC & 0.40 \\
\hline
\end{tabular}

$\mathrm{pH} \mathrm{NaOH}-\mathrm{AAS}$ pastes with respect to 11.7-pH NaOH-AAS and OPC pastes. Immediately after mixing, the pastes were placed in a Haake Rheowin Pro RV1 viscosimeter having concentric cylinders with roughened surfaces coaxial rotor, and subjected to a 5-cycle measuring procedure (see Fig. 2). During each five-minute cycle, the shear rate was first kept constant at $200 \mathrm{~s}^{-1}$ for 2 min of preshearing, then ramped from 0 to $10 \mathrm{~s}^{-1}$ in $1 \mathrm{~min}$, from 10 to $200 \mathrm{~s}^{-1}$ in $1 \mathrm{~min}$ and finally from 200 to $50 \mathrm{~s}^{-1}$ in $1 \mathrm{~min}$. The second cycle was begun immediately after the first and so on successively, with cycles ending 5 , $10,15,20$ and 25 min after mixing.

\section{Results and discussion}

3.1. Determination of superplasticizer adsorption isotherms on AAS and OPC pastes and the effect of superplasticizers on the zeta potential of AAS and OPC suspensions

The adsorption isotherms for the three superplasticizers on 11.7and 13.6-pH NaOH-AAS and OPC are shown in Fig. 3. In 11.7- and 13.6$\mathrm{pH} \mathrm{NaOH}-\mathrm{AAS}$ pastes the adsorption of melamine-based admixture is linear at initial concentrations of admixture lower than $6 \mathrm{mg}$ superplasticizer/g slag (see Fig. 2a). At higher dosages melamine-based admixture was adsorbed gradually reaching a maximum of adsorbed admixture of approximately $11 \mathrm{mg}$ superplasticizer adsorbed/g slag. By contrast, Fig. 2a also shows that the melamine-based admixture was linearly adsorbed onto OPC pastes at all concentrations of the superplasticizer and that OPC pastes were able to adsorb much greater amounts of admixture than the respective AAS materials.

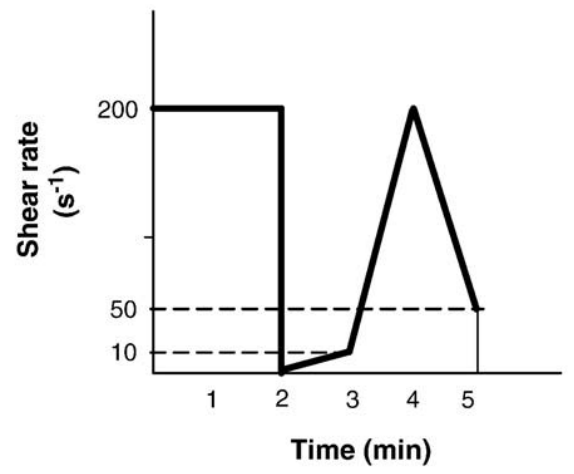

Fig. 2. Shear rate evolution in the rheological test conducted on the pastes.
Adsorption of the naphthalene-based admixture on 11.7- and 13.6pH NaOH-AAS pastes (see Fig. 2b), in turn, was linear at admixture initial concentrations lower than $5 \mathrm{mg}$ superplasticizer/g slag. At these low concentrations of admixtures, Fig. 2a,b shows that the slag adsorbed a lower amount of naphthalene admixture than melamine, which means that the affinity of slag for the melamine was higher. Upward of $5 \mathrm{mg}$ superplasticizer/g slag the naphthalene was adsorbed progressively, to a maximum of $5.5 \mathrm{mg}$ superplasticizer adsorbed $/ \mathrm{g}$ slag. Here also, much more naphthalene-based admixture was

a)

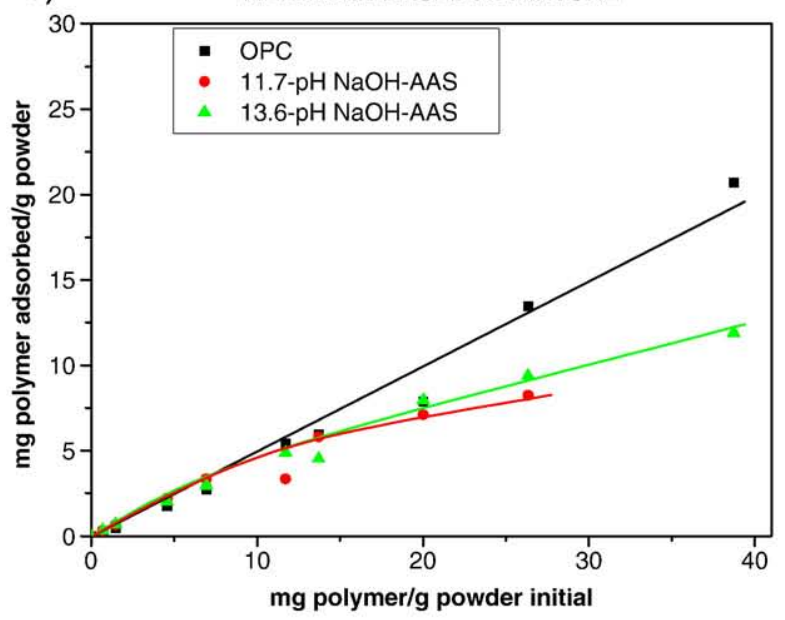

b) NAPHTHALENE-BASED ADMIXTURE

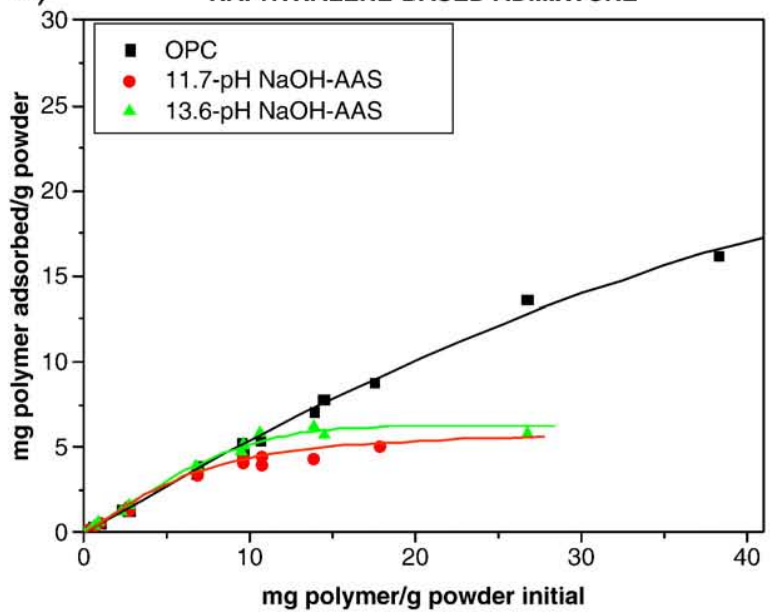

C) VINYL COPOLYMER ADMIXTURE

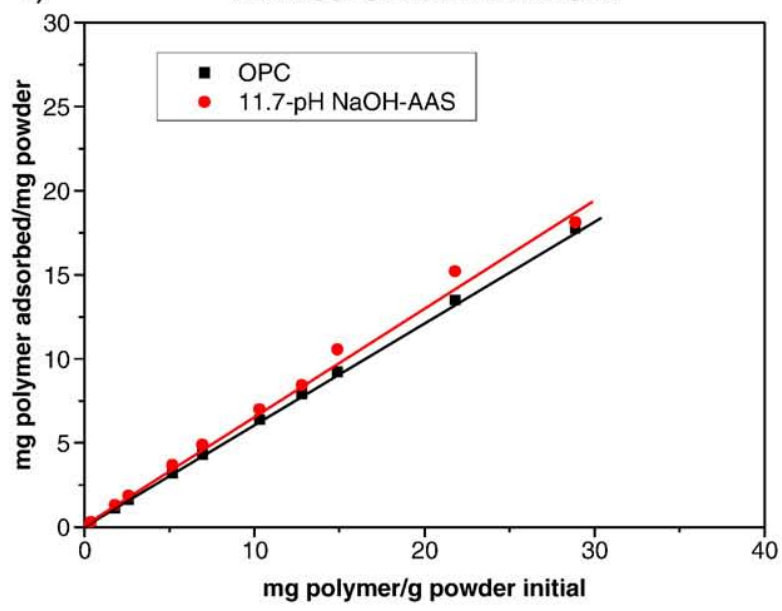

Fig. 3. Superplasticizer adsorption isotherms on OPC and AAS pastes. 


\section{a) 11.7-pH NaOH-AAS + MELAMINE BASED ADMIXTURE}

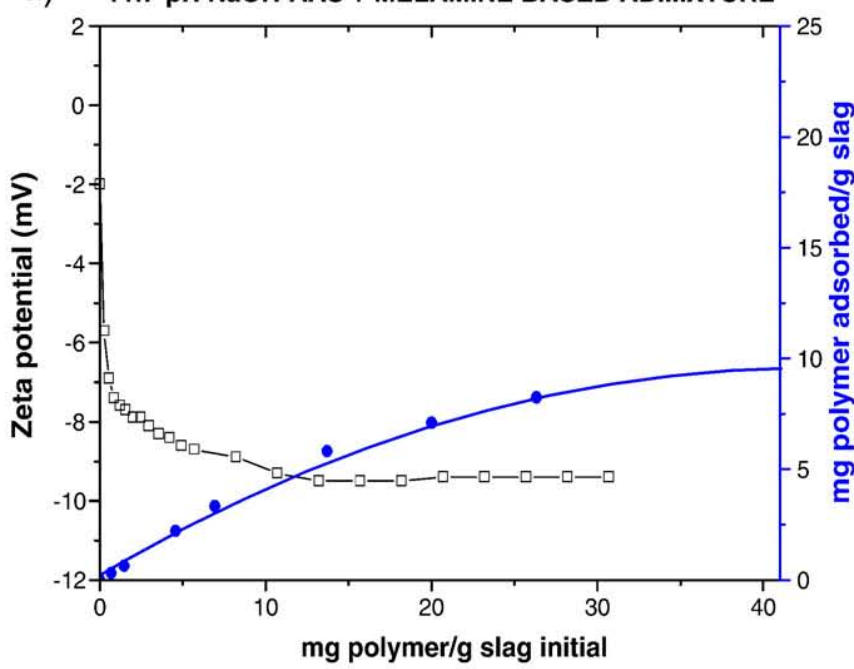

b) 11,7-pH NaOH-AAS + NAPHTHALENE-BASED ADMIXTURE

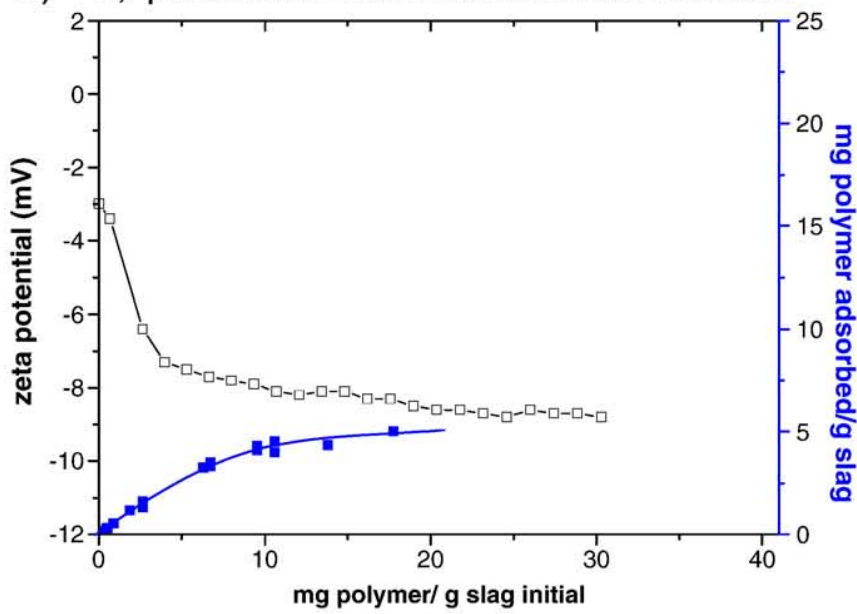

C) 11.1-pH + NaOH-AAS + VINYL COPOLYMER ADMIXTURE

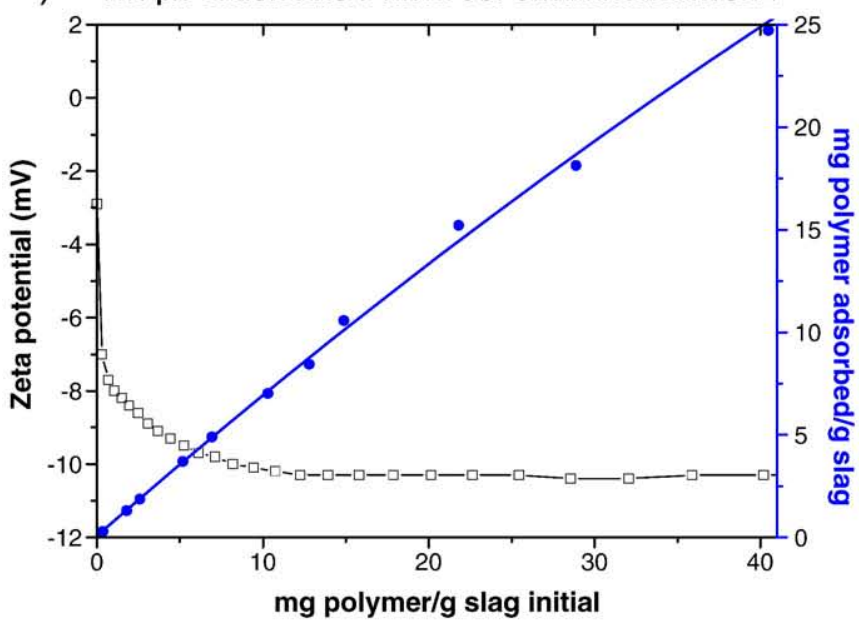

Fig. 4. Variation in the zeta potential in $11.7-\mathrm{pH} \mathrm{NaOH}-\mathrm{AAS}$ slag pastes adsorbing superplasticizers.

adsorbed onto the OPC than onto the AAS pastes. In OPC pastes, adsorption of this admixture was linear for initial concentrations of admixture lower than $26 \mathrm{mg}$ superplasticizer/g cement and the maximum amount of naphthalene-based superplasticizer adsorbed came to approximately $16 \mathrm{mg}$ superplasticizer adsorbed/g cement.
From adsorption isotherms of naphthalene- and melamine-based admixtures we can conclude that their adsorption on AAS pastes is independent of the $\mathrm{pH}$ of the solution used.

The OPC pastes adsorbed twice as much superplasticizer as the AAS pastes. Since the two binders have similar specific surfaces (Table 1 ), this factor cannot explain the differences in behaviour. The two binders are observed to have slightly different zeta potentials as a result of the differences in their chemical and mineralogical composition. The

a)
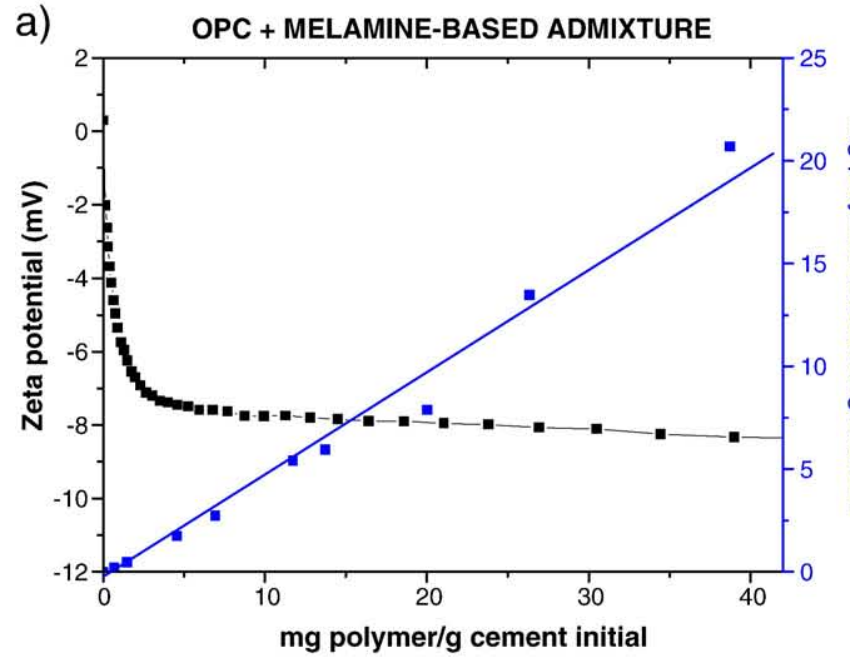

b) OPC + NAPHTHALENE-BASED ADMIXTURE
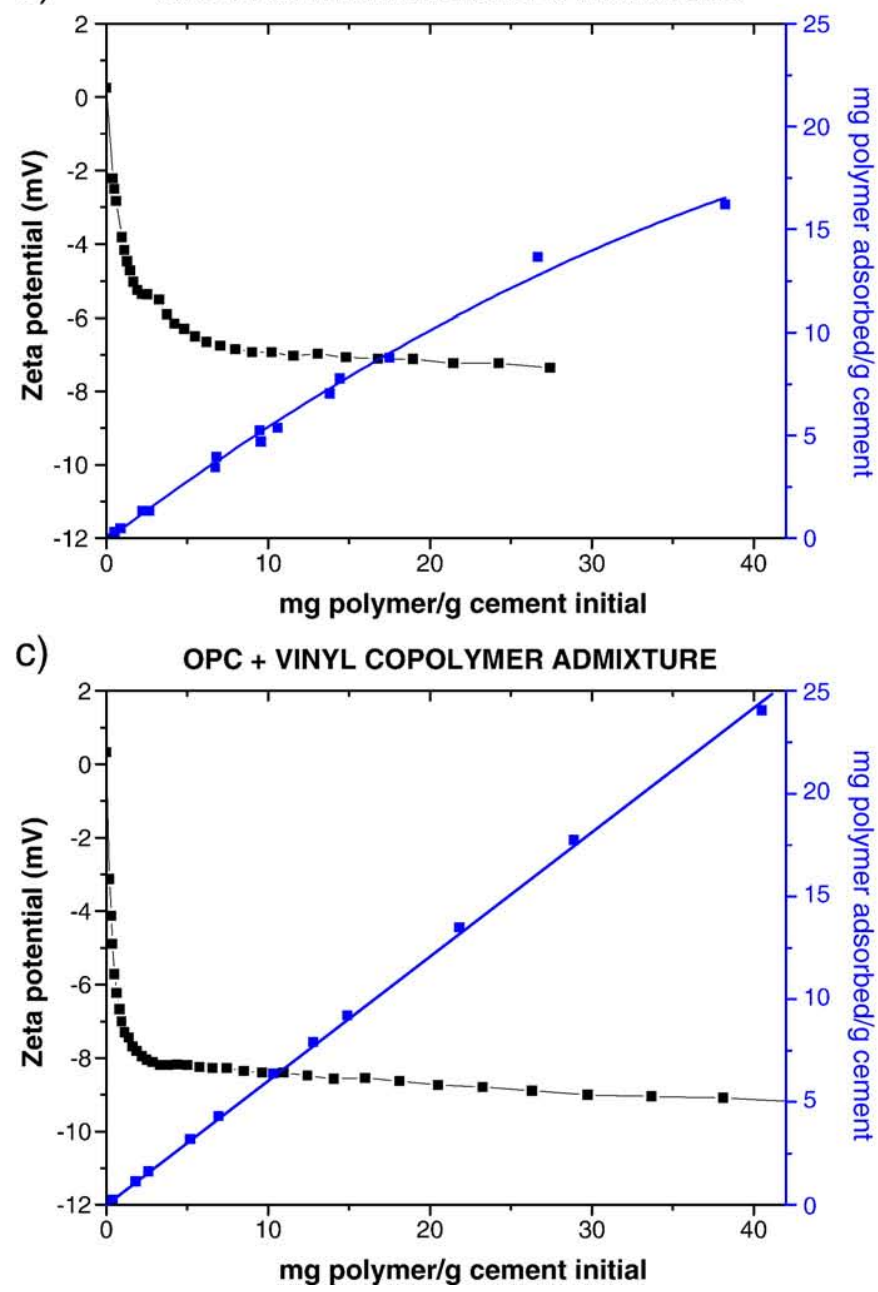

Fig. 5. Variation in the zeta potential in OPC pastes adsorbing superplasticizers. 


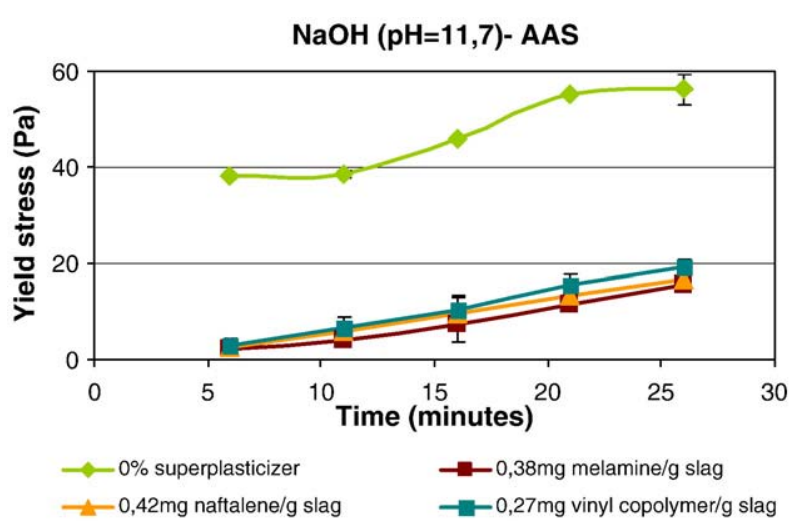

Fig. 6. Yield stress evolution in 11.7-pH NaOH-AAS pastes containing superplasticizers.

zeta potential of AAS suspensions is slightly negative (approximately $-2 \mathrm{mV}$, see Fig. 4), while the zeta potential of OPC is slightly positive (approximately $+0.5 \mathrm{mV}$, see Fig. 5). Inasmuch as the admixtures are adsorbed as a result of the interaction between their anion group and the positively charged sites on cement and slag particles, the more positive the zeta potential, the greater the number of adsorption sites may be expected. This would explain at least in part the higher capacity of OPC to adsorb the admixtures. In OPC pastes the superplasticizers are not only absorbed on the mineralogical anhydrous phases, but a certain proportion of the admixtures can also be consumed by $\mathrm{C}_{3} \mathrm{~A}$ forming an intercalated organo-mineral phase $[11,21]$ and additionally, early hydration of OPC forms ettringite which also adsorbs superplasticizer due to its positive zeta potential [22]. Therefore, the adsorption curves of the superplasticizers on OPC pastes shown in Fig. 3a,b correspond to the quantity of admixture consumed (adsorbed and intercalated) by the Portland cement. The AAS systems do not contain $C_{3} A$ and $C_{3} A$ hydrated phases so that the superplasticizers are not consumed by the formation of an organo-mineral phase explaining the lower quantity of admixture really adsorbed by AAS pastes compared to OPC pastes.

The vinyl copolymer adsorption was a linear function of superplasticizer concentration onto both OPC and 11.7-pH NaOH-AAS pastes at all the admixture concentrations, without any trend towards a plateau value at any time during the test (see Fig. 3c). These results could be explained by the fact that this admixture possibly precipitates on the surface of the particles in highly alkaline media; therefore the adsorption curves of this admixture on 13.6-pH NaOH-AAS were not determined.

Fig. 4 shows the incorporation of the admixtures to the 11.7-pH $\mathrm{NaOH}-\mathrm{AAS}$ suspensions induces a shift to more negative values. Similar shift of the zeta potential of blast furnace slag in presence of surfactants were observed by Nägele and Schneider [23]. The greatest variation in the zeta potential was observed at admixture dosages under $5 \mathrm{mg}$ superplasticizer/g initial slag and the zeta potential variation arrived at a plateau value at dosages around $15 \mathrm{mg}$ superplasticizer/g slag. Fig. 4 also shows that zeta potential induced by the vinyl copolymer was slightly more negative $(-10 \mathrm{mV})$ than the values induced by the melamine $(-9 \mathrm{mV})$ and naphthalene-based $(-8 \mathrm{mV})$ admixtures. Similar effect of the superplasticizers on the zeta potential of OPC suspensions was observed (see Fig. 5). In this case, dosages lower than $5 \mathrm{mg}$ superplasticizer/g initial slag also produce the highest decrease of the zeta potential.

\subsection{Study of the effect of admixtures on AAS and OPC paste rheology}

The influence of the superplasticizers on the rheological parameters (yield stress and plastic viscosity) of AAS and OPC pastes was evaluated. The dosages that gave a maximum reduction in yield stress without promoting segregation was determined when fluidizing properties were observed.

Figs. 6-8 show that in the absence of the admixture, the yield stress values for the 11.7-pH NaOH-AAS pastes were slightly higher than the values for OPC pastes, while the 13.6-pH NaOH-AAS pastes exhibited the lowest yield stress values. These figures also show the effect of the adsorption of the admixtures on the yield stress, associated with the dispersion of agglomerated particles [24]. Further to these results, the effect of the admixtures depends directly on their type and dosage as well as of the binder used. In the case of the AAS pastes the yield stress also depends on the $\mathrm{pH}$ of the alkaline activator solution, contrary to the adsorption isotherms. Additionally, an increase of the yield stress of OPC and AAS pastes was observed as the test progresses due to the partial lost of the fluidizing properties of the superplasticizers with the time and the formation of new hydration products.

Fig. 6 shows that in 11.7-pH NaOH-AAS pastes even very small dosages (lower than $10 \%$ of the adsorption plateau) of the admixtures lower yield stress by up to $92 \%$ confirming their chemical stability and
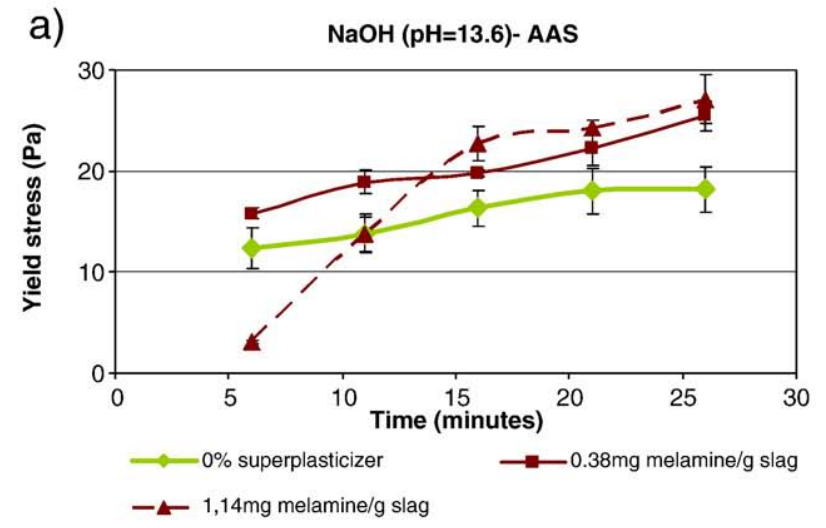

b) 13.6-pH NaOH- AAS

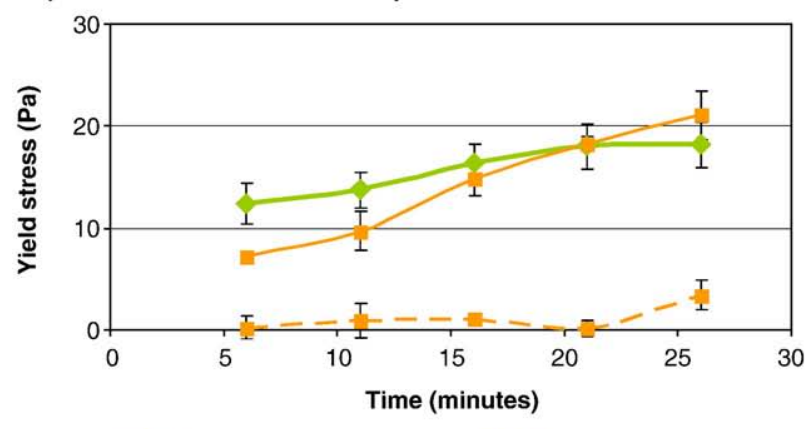

$\longrightarrow$
-

C) 13.6-pH NaOH- AAS

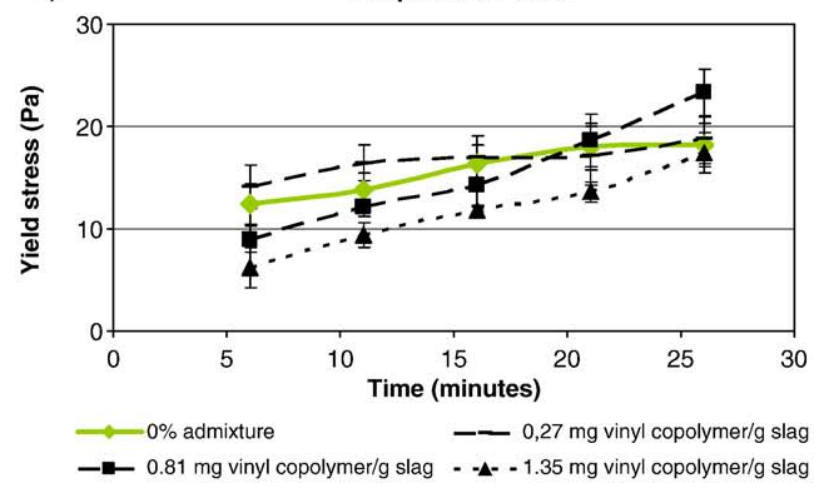

Fig. 7. Yield stress evolution in 13.6-pH NaOH-AAS pastes containing superplasticizers. 
a)

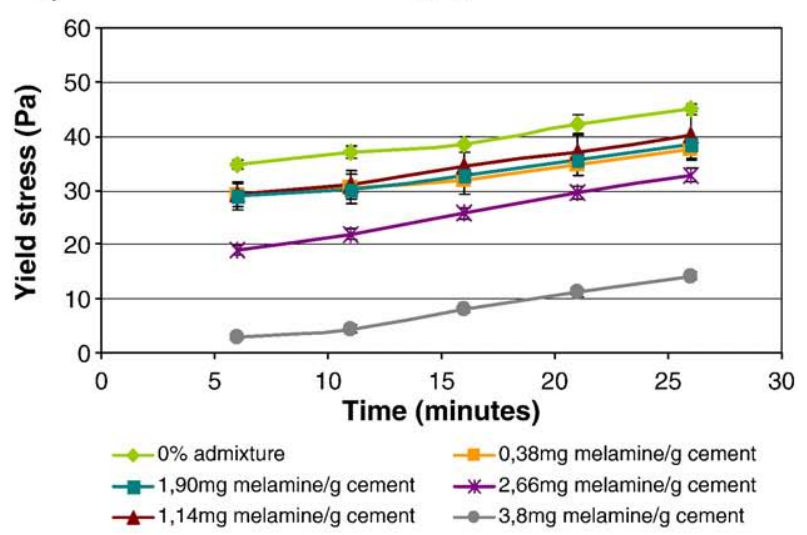

b)

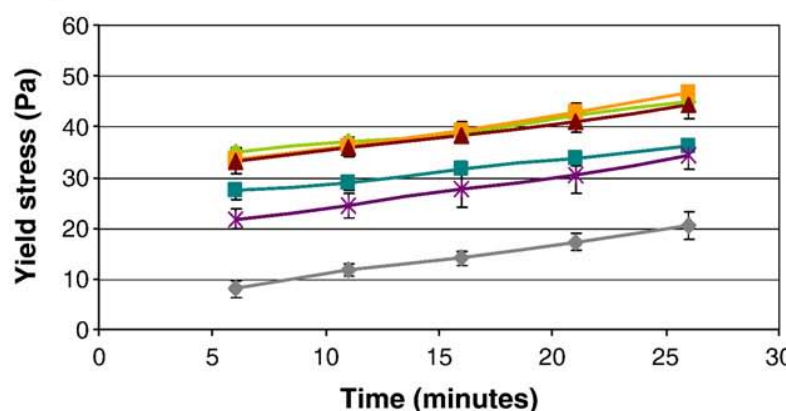

Time (minutes)

$-0,42 \mathrm{mg}$ naphthalene/g cement $-0 \%$ admixture $\leftarrow 1,26 \mathrm{mg}$ naphthalene/g cement $\rightarrow 4,20 \mathrm{mg}$ naphthalene/g cement

c)

OPC
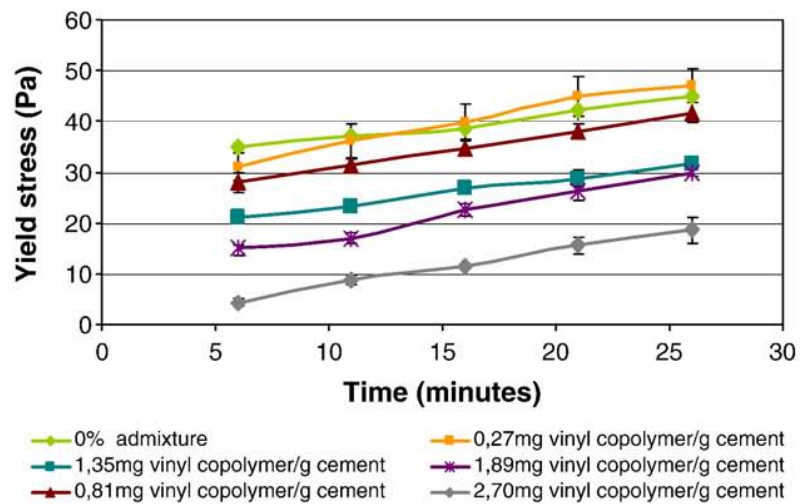

Fig. 8. Yield stress evolution in OPC pastes containing superplasticizers.

excellent fluidizing properties in this alkaline medium [25]. In this series of pastes, a dosage of $0.27 \mathrm{mg}$ of vinyl copolymer/g slag produces the same decrease in yield stress as higher dosages of the melamine- and naphthalene-based admixtures ( 0.38 and $0.42 \mathrm{mg}$ superplasticizer $/ \mathrm{g}$ slag, respectively). Two possible explanations are 1) adsorption of this admixture leads to a more negative zeta potential than adsorption of the other two admixtures (see Fig. 4); 2 ) the vinyl copolymer exhibits higher molecular weight (see Table 2). The steric contribution to particle dispersion is closely linked to the adsorbed layer thickness which in turn is linked to the molecular weight. These two elements provide substantially larger electrosteric repulsion generated between the slag particles by the vinyl copolymer and the concomitant reduction in yield stress. To better understand the relative importance of the steric and electrostatic contributions more detailed studies using the recent theoretical model (YODEL) [24] and further characterization of the adsorbed thickness for example by AFM are needed.

In 13.6-pH NaOH-AAS pastes, naphthalene-based admixture is the only superplasticizer observed to produce a significant decrease in yield stress (98\% - see Fig. 7b). In this case although the slag is able to adsorb more than $5.5 \mathrm{mg}$ naphthalene/g slag, dosages as low as $1.26 \mathrm{mg}$ naphthalene/g slag are observed to induce the maximum reduction in yield stress and consequently the maximum rise in paste flowability, while higher dosages of this admixture caused paste segregation. Consequently, the presence of naphthalene admixture in $13.6-\mathrm{pH} \mathrm{NaOH}-$ AAS systems should allow us to decrease significantly the $1 / \mathrm{s}$ and as previous studies have demonstrated there is also an improvement in the mechanical and durable properties of these alkaline-cements [18].

The effective fluidization of 13.6-pH NaOH-AAS pastes by the naphthalene superplasticizer is due to its chemical stability in such extremely alkaline media [19]. The melamine-based and vinyl copolymer superplasticizers, however, do not substantially improve flowability in 13.6-pH NaOH-AAS pastes (see Fig. 7a,c), even though greater amounts of these than of the naphthalene-based superplasticizer are adsorbed. The presence of $1.14 \mathrm{mg}$ melamine/g slag and $1.35 \mathrm{mg}$ vinyl copolymer/g slag lowered initially yield stress significantly, although this effect disappeared after only 11 min under these conditions. This failure of melamine-based and vinyl copolymer admixtures in 13.6-pH NaOH-AAS pastes is attributed to their chemical instability in such extremely basic media which conduct to the loss of their fluidizing properties [18]. This chemical instability leads to lower molecular weight fragments and although these fragments may still adsorbs and induce an electrostatic repulsion, the steric contribution will be significantly reduced.

In OPC pastes all three admixtures show significant fluidizing capacity (see Fig. 8). For the melamine-based admixture, yield stress decreased with increasing dosages (see Fig. 8a), dropping by a maximum of $91 \%$ at a dosage of $3.8 \mathrm{mg}$ melamine/g cement. Fig. $7 \mathrm{~b}$ shows that the presence of the naphthalene-based admixture lowered the yield stress in cement pastes by up to $77 \%$ at dosages of $4.20 \mathrm{mg}$ naphthalene/g cement. Finally, the greatest decline in cement paste yield stress prompted by the presence of the vinyl copolymer (87\%) was recorded for a smaller dosage of this admixture than naphthalene and melamine-based admixtures: $2.70 \mathrm{mg}$ of pure vinyl copolymer/g cement (see Fig. 8c). A comparison of the effect of the superplasticizers on yield stress in AAS and OPC pastes shows that the dosages required to attain similar reductions in this parameter in the two binders are ten-fold higher in OPC pastes than in 11.7-pH NaOH-AAS. Similarly, in 13.6-pH NaOH-AAS pastes, the amount of naphthalene-based admixture required to arrive at the highest decrease of yield stress is 3 times lower than in OPC pastes. Consequently, despite the lower adsorption on slag compared to cement particles, the amount of admixture present in the former suffices to produce greater inter-particle electrosteric repulsion and greater decreases in yield stress than generated by much larger amounts of admixture in OPC cements, where the

11.7-pH NaOH- AAS

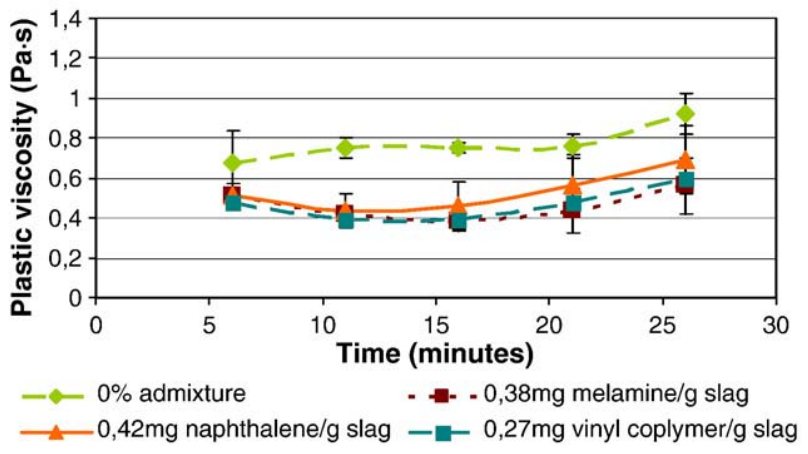

Fig. 9. Plastic viscosity evolution in 11.7-pH NaOH-AAS pastes containing superplasticizers. 
a)

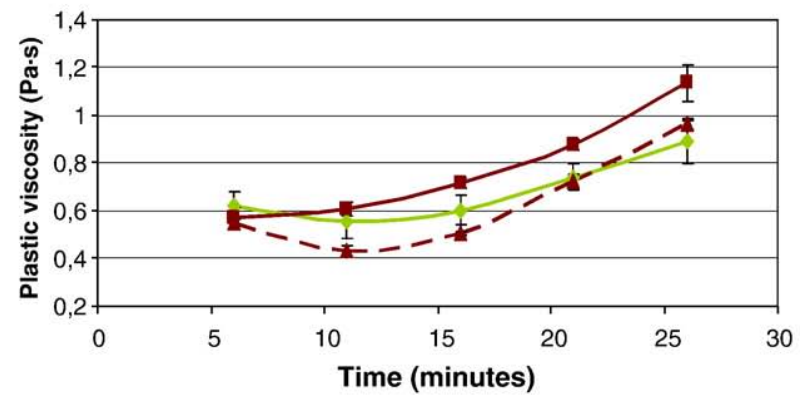

$\longrightarrow 0 \%$ admixture $\quad \longrightarrow 0,38 \mathrm{mg}$ melamine/g slag

$-\longleftarrow 1,14 \mathrm{mg}$ melamine/g slag

b)

13.6-pH NaOH- AAS

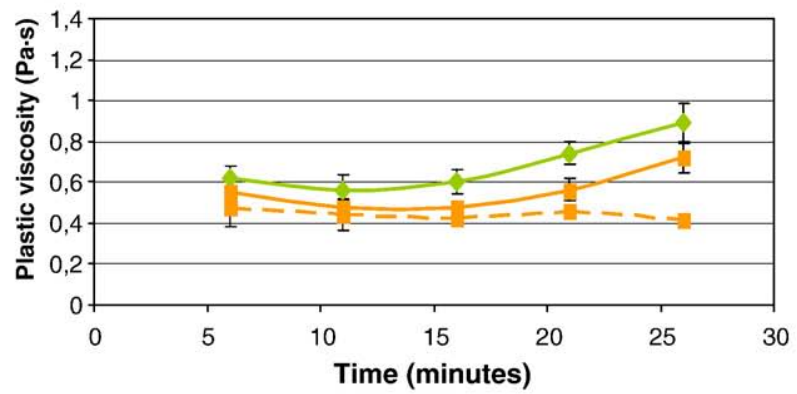
$-0 \%$ admixture
$-1,26 \mathrm{mg}$ naphthalene/g slag

c)

13.6-pH NaOH- AAS

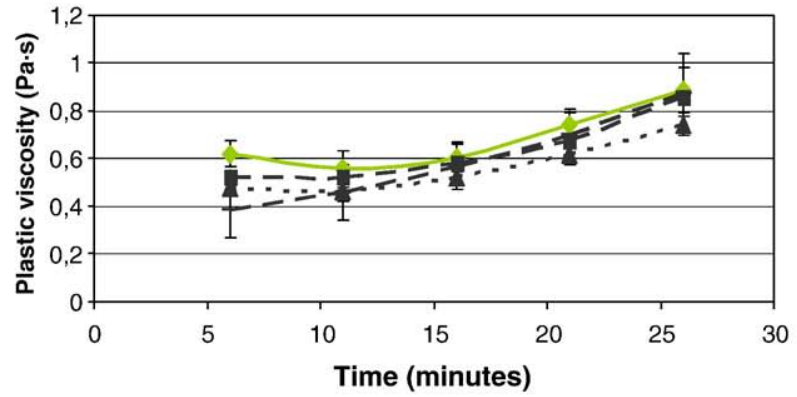

$\rightarrow-0 \%$ admixture $\quad-0,27 \mathrm{mg}$ vinyl copolymer/g slag

- $0.81 \mathrm{mg}$ vinyl copolymer/g slag $\cdot-\Delta-1,35 \mathrm{mg}$ vinyl copolymer/g slag

Fig. 10. Plastic viscosity evolution in 13.6-pH NaOH-AAS pastes containing superplasticizers.

admixture is probably partly consumed and will not contribute to the electrosteric repulsion between cement particles [11]. However, further studies must be carried out to support this statement.

With respect to the influence of the superplasticizers on the plastic viscosity of AAS cements, Figs. 9 and 10 shows that the presence of the three admixtures lowers the plastic viscosity in the 11.7-pH NaOH-AAS pastes and only the naphthalene-based admixture in 13.6-pH NaOHAAS pastes, due to the adsorption-mediated decrease in the size and number of agglomerated particles. In 11.7-pH $\mathrm{NaOH}$-activated slag pastes the superplasticizer admixtures decrease the plastic viscosity by up to $48 \%$ at dosages of $0.38,0.42$ and $0.27 \mathrm{mg}$ superplasticizer/g slag respectively (see Fig. 9), while in 13.6-pH NaOH-activated slag pastes, the presence of $1.26 \mathrm{mg}$ of naphthalene/g slag (see Fig. 10b) lowered the plastic viscosity by $23 \%$, confirming the chemical stability of this admixture in this high alkaline media. The melamine-based and vinyl copolymer admixtures, on the contrary, only induced a slight decrease of plastic viscosity during the first $10 \mathrm{~min}$ of the rheological test in 13.6-pH $\mathrm{NaOH}$-activated slag pastes. However after $10 \mathrm{~min}$, no fluidizing properties of these admixtures are observed and the vinyl copolymer admixture even induces an increase of the plastic viscosity, although the reason of this increase is not clear (see Fig. 10c).

In OPC pastes, the melamine- and naphthalene-based admixtures lowered plastic viscosity (see Fig. 11a,b, respectively) during the first 5 min of the test. The vinyl copolymer admixture did not induce a decrease in plastic viscosity which became more pronounced as a function of time (by approximately 62\%) when the amount of admixture added was larger (see Fig. 11c).

\section{Conclusions}

1. Adsorption of melamine-based, naphthalene-based and vinyl copolymer admixtures on AAS pastes is independent of the $\mathrm{pH}$ of the

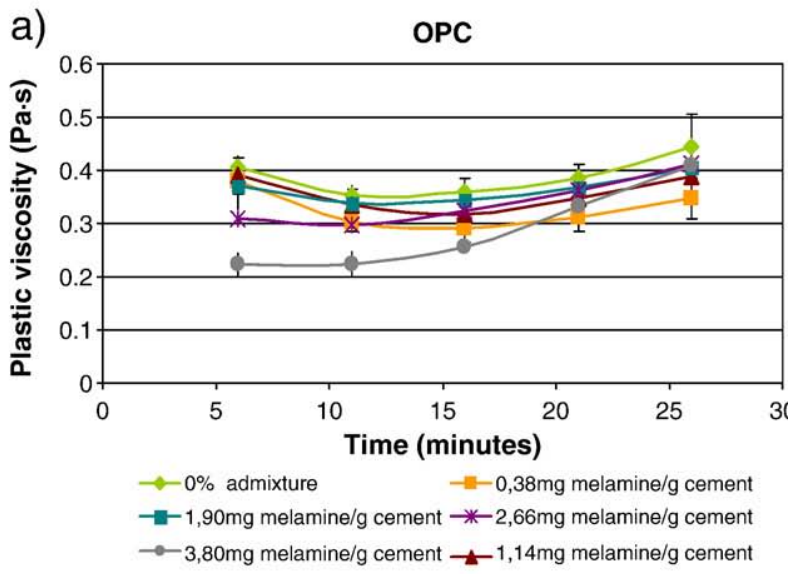

b)

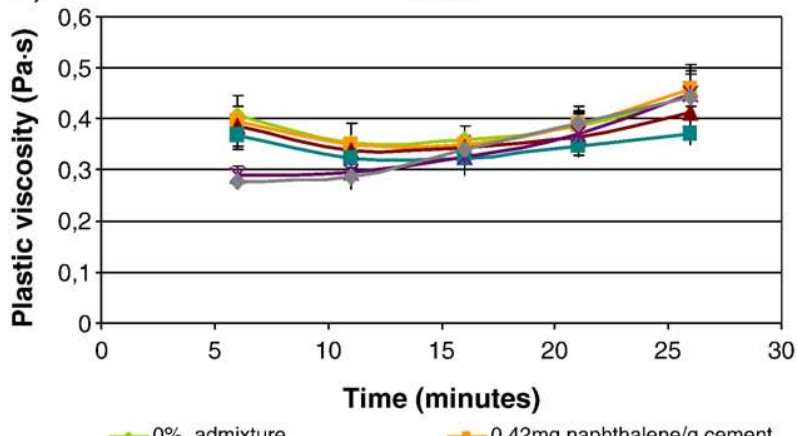

$\rightarrow-0 \%$ admixture $\quad-0,42 \mathrm{mg}$ naphthalene/g cement $--2,10 \mathrm{mg}$ naphthalene/g cement $\rightarrow-2,94 \mathrm{mg}$ naphthalene/g cement $\leftarrow 1,26 \mathrm{mg}$ naphthalene/g cement $\rightarrow-4,20 \mathrm{mg}$ naphthalen/g cement

c) $\mathrm{OPC}$

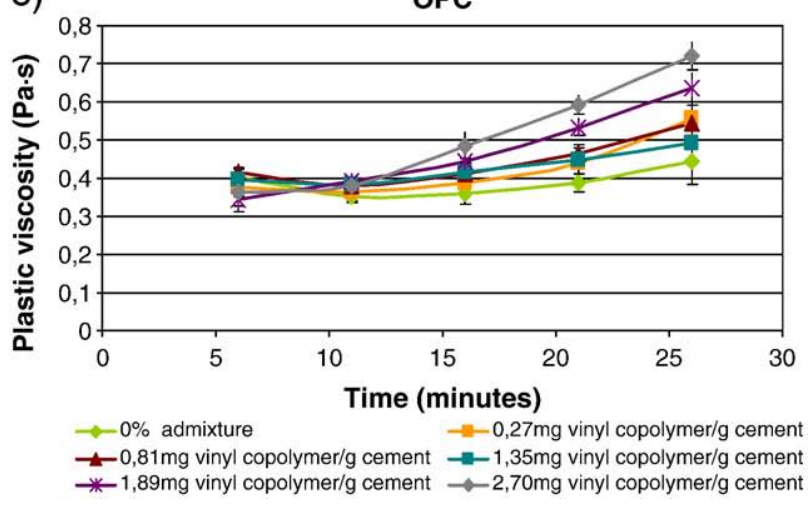

Fig. 11. Plastic viscosity evolution in OPC pastes containing superplasticizers. 
solution used and significantly lower than on OPC pastes by a factor between 3 and 10 .

The zeta potential of $11.7-\mathrm{pH} \mathrm{NaOH}$ AAS suspensions studied is slightly more negative (approximately $-2 \mathrm{mV}$ ) than the zeta potential of OPC suspensions (approximately $+0.5 \mathrm{mV}$ ), which may partially explain the different adsorption behaviour of both cements.

2. The effect of the admixtures on the rheological parameters depends directly on the type and dosage of the superplasticizer as well as on the binder used and, in the case of the alkali-activated slag pastes, also on the $\mathrm{pH}$ of the alkaline activator solution.

3. The dosages of the superplasticizers required to attain similar reduction in the yield stress are ten-fold higher for Portland cement than for $11.7-\mathrm{pH} \mathrm{NaOH}$-activated slag pastes suggesting that a high proportion of these admixtures are consumed in organo-mineral phases and do not contribute to fluidification. Vinyl copolymer admixture induces the highest reduction of the yield stress in 11.7$\mathrm{pH} \mathrm{NaOH}$-activated slag pastes. This was attributed to both the electrostatic repulsion from a higher induced zeta potential and a thicker adsorbed layer related to its higher molecular weight.

4. The only admixture observed to affect the rheological parameters in 13.6-pH NaOH-activated slag is the naphthalene-based admixture due to its structural stability in such extremely alkaline media. Dosages as low as $1.26 \mathrm{mg}$ naphthalene/g slag are observed to induce the maximum reduction in yield stress (98\%).

\section{Acknowledgements}

Funding for project CTM2004-06619-CO2-01 was provided by the Spanish Ministry of Science and Technology (MCyT). The authors wish to thank Carlos Morais for help with the zeta potential measurements and to Dr. Carmen Peinado and Mario Luzón for help with the GPC of the admixtures. M. Palacios worked under a postdoctoral contract awarded by the CSIC.

\section{References}

[1] T. Bakharev, J.G. Sanjayan, Y.B. Cheng, Alkali activation of Australian slag cements, Cem Concr Res 29 (1999) 113-120.

[2] C. Shi, Strength, pore structure and permeability of alkali-activated slag mortars, Cem Concr Res 26 (1996) 1789-1799.

[3] A. Fernández-Jiménez, F. Puertas, Effect of activator mix on the hydration and strength behaviour of alkali-activated slag cements, Adv Cem Res 15 (3) (2003) 129-136.
[4] T. Bakharev, J.G. Sanjayan, Y.B. Cheng, Resistance of alkali-activated slag concrete to acid attack, Cem Concr Res 33 (2003) 1607-1611.

[5] F. Puertas, R. Mejía de Gutierrez, A. Fernández-Jiménez, S. Delvasto, J. Maldonado, Alkaline cement mortars. Chemical resistance to sulfate and seawater attack, Mater Constr 52 (267) (2002) 55-71.

[6] J. Björnstrom, S. Chandra, Effect of superplasticizers on the rheological properties of cements, Mat. Struct. 36 (2003) 685-692.

[7] F. Puertas, H. Santos, M. Palacios, S. Martínez-Ramírez, Polycarboxylate superplasticizer admixtures. Effect on hydration, microstructure and rheological behaviour in cement pastes, Adv Cem Res 17 (2005) 77-89.

[8] M.Y.A. Mollah, W.J. Adams, R. Schennach, D.L. Cocke, A review of cementsuperplasticizer interactions and their models, Adv Cem Res 12 (2000) 153-161.

[9] Y. Houst, P. Bowen, A. Siebold, Some basic aspects of the interaction between cement and superplasticizers, in: R.K. Dhir, P.C. Hewlett, L.J. Csetenvi (Eds.), Innovations and Developments in Concrete Materials and Construction, 2002. 225-234.

[10] M.M. Alonso, M. Palacios, F. Puertas, A.G. de la Torre, M.A.G. Aranda, Effect of polycarboxylate admixture structure on cement paste rheology, Mater Constr 57 (286) (2007) 65-81.

[11] R.J. Flatt, Y. Houst, A simplified view on chemical effects perturbing the action of superplasticizers, Cem Conc Res 31 (2001) 1169-1176.

[12] K. Yoshioka, W. Tazawa, K. Hawai, T. Enohata, Adsorption characteristics of superplasticizers on cement component minerals, Cem Concr Res 32 (2002) 1507-1513.

[13] K. Yamada, S. Ogawa, S. Hanehara, Controlling of the adsorption and dispersing force of polycarboxylate-type superplasticizer by sulfate ion concentration in aqueous phase, Cem Concr Res 31 (2001) 375-383.

[14] U. Mäder, I. Schober, Performance of blends of polycarboxylate polymers in different cements, XI (ICCC) Durban, South Africa, 2003. 504-513.

[15] S. Chandra, J. Björnström, Influence of cement and superplasticizers type and dosage on the fluidity of cement mortars. Part I, Cem Concr Res 32 (2002) 1605-1611.

[16] U. Mäder, I. Schober, F. Wombacher, D. Ludirdja, Polycarboxylate polymers and blends in different cements, Cem, Concr Aggreg 26 (2004) 110-114.

[17] T. Bakharev, J.G. Sanjayan, Y.B. Cheng, Effect of admixtures on properties of alkaliactivated slag concrete, Cem Concr Res 30 (2000) 1367-1374.

[18] M. Palacios, F. Puertas, Effect of superplasticizer and shrinkage-reducing admixtures on alkali-activated slag pastes and mortars, Cem Concr Res 35 (2005) 1358-1367.

[19] M. Palacios, F. Puertas, Stability of superplasticizer and shrinkage-reducing admixtures in high basic media, Mater Constr 54 (276) (2004) 65-68.

[20] F. Perche, "Adsorption de polycarboxylates et de lignosulfonates sur poudre modèle et ciments". Thése EPFL No 3041, Lausanne. 2004. (Can be downloaded from: http://library.epfl.ch/en/theses/?nr=3041).

[21] J. Plank, Z. Dai, N. Zouaoui, D. Vlad, Intercalation of polycarboxylate superplasticizers into $\mathrm{C}_{3} \mathrm{~A}$ hydrate phases, in: V.M. Malhotra (Ed.), Eighth CANMET/ACI Conference on Superplasticizers in Concrete, ACI, Sorrento, SP, vol. 239-14, 2006. 201-214.

[22] J. Plank, C. Hirsch, Impact of zeta potential of early cement hydration phases on superplasticizer adsorption, Cem Concr Res 37 (2007) 537-542.

[23] E. Nägele, U. Schneider, The zeta-potential of blast furnace slag and fly ash, Cem Concr Res 19 (1989) 811-820.

[24] R.J. Flatt, P. Bowen, Yield stress of multimodal powder suspensions: an extension of the YODEL (Yield Stress Model), J Am Ceram Soc 90 (4) (2007) 1038-1044.

[25] M. Palacios, Efecto de aditivos orgánicos en las propiedades de los cementos y morteros de escoria activada alcalinamente. PhD. Universidad Autónoma de Madrid. 2006. 